F2-2012-279017) and NEUROKINE network (EU Framework 7 ITN project).

Address correspondence to: Gianvito Martino, Neuroimmunology Unit, Institute of Experimental Neurology (INSpe), Division of Neuroscience, San Raffaele Scientific Institute, Via Olgettina 58, 20132 Milan, Italy. Phone: 39.02.2643.4853; Fax: 39.02. 2643.4855; E-mail: martino.gianvito@hsr.it.

1. Alvarez-Buylla A, Lim DA. For the long run: main taining germinal niches in the adult brain. Neuron. 2004;41(5):683-686.

2. Zhao C, Deng W, Gage FH. Mechanisms and functional implications of adult neurogenesis. Cell. 2008;132(4):645-660.

3. Kitamura T, et al. Adult neurogenesis modulates the hippocampus-dependent period of associative fear memory. Cell. 2009;139(4):814-827.

4. Spalding KL, et al. Dynamics of hippocampal neurogenesis in adult humans. Cell. 2013;153(6):1219-1227.

5 . Lu Z, et al. Phagocytic activity of neuronal progenitors regulates adult neurogenesis. Nat Cell Biol. 2011;13(9):1076-1083.

6. Mosher KI, et al. Neural progenitor cells regulate microglia functions and activity. Nat Neurosci. 2012; 15(11):1485-1487.

7. Mohammad MG, et al. Immune cell trafficking from the brain maintains CNS immune tolerance. JClin Invest. 2014;124(3):1228-1241.

8. Sawamoto K, et al. New neurons follow the flow of cerebrospinal fluid in the adult brain. Science. 2006;311(5761):629-632.

9. Butti E, et al. Subventricular zone neural progenitors protect striatal neurons from glutamatergic excitotoxicity. Brain. 2012;135(pt 11):3320-3335.

10. Stock K, et al. Neural precursor cells induce cell death of high-grade astrocytomas through stimulation of TRPV1. Nat Med. 2012;18(8):1232-1238.

11. Martino G, Pluchino S. The therapeutic potential of neural stem cells. Nat Rev. 2006;7(5):395-406.

12. Ourednik J, Ourednik V, Lynch WP, Schachner M, Snyder EY. Neural stem cells display an inherent mechanism for rescuing dysfunctional neurons. Nat Biotechnol. 2002;20(11):1103-1110.

13. Pluchino $\mathrm{S}$, et al. Injection of adult neurospheres induces recovery in a chronic model of multiple sclerosis. Nature. 2003;422(6933):688-694.

14. Bacigaluppi M, et al. Delayed post-ischaemic neuroprotection following systemic neural stem cell transplantation involves multiple mechanisms. Brain. 2009;132(pt 8):2239-2251.

15. Menn B, Garcia-Verdugo JM, Yaschine C, Gonzalez-Perez O, Rowitch D, Alvarez-Buylla A. Origin of oligodendrocytes in the subventricular zone of the adult brain. J Neurosci. 2006;26(30):7907-7918.

16. Etxeberria A, Mangin JM, Aguirre A, Gallo V. Adultborn SVZ progenitors receive transient synapses during remyelination in corpus callosum. Nat Neurosci. 2010;13(3):287-289.
17. Benner EJ, et al. Protective astrogenesis from the SVZ niche after injury is controlled by Notch modulator Thbs4. Nature. 2013;497(7449):369-373.

18. Sabelstrom $\mathrm{H}$, et al. Resident neural stem cells restrict tissue damage and neuronal loss after spinal cord injury in mice. Science. 2013;342(6158):637-640.

19. Parent JM, von dem Bussche N, Lowenstein DH. Prolonged seizures recruit caudal subventricular zone glial progenitors into the injured hippocampus. Hippocampus. 2006;16(3):321-328.

20. Parent JM, Yu TW, Leibowitz RT, Geschwind DH, Sloviter RS, Lowenstein DH. Dentate granule cell neurogenesis is increased by seizures and contributes to aberrant network reorganization in the adult rat hippocampus. J Neurosci. 1997;17(10):3727-3738.

21. Tavazoie $M$, et al. A specialized vascular niche for adult neural stem cells. Cell Stem Cell. 2008; 3(3):279-288

22. Mirzadeh Z, Merkle FT, Soriano-Navarro M, GarciaVerdugo JM, Alvarez-Buylla A. Neural stem cells confer unique pinwheel architecture to the ventricular surface in neurogenic regions of the adult brain. Cell Stem Cell. 2008;3(3):265-278

23. Rolls A, et al. Toll-like receptors modulate adult hippocampal neurogenesis. Nat Cell Biol. 2007; 9(9):1081-1088

24. Koos T, Tepper JM. Inhibitory control of neostriatal projection neurons by GABAergic interneurons. Nat Neurosci. 1999;2(5):467-472.

25. Pluchino $S$, et al. Persistent inflammation alters the function of the endogenous brain stem cell compartment. Brain. 2008;131(pt 10):2564-2578.

\title{
Dangerous liaisons: flirtations between oncogenic BRAF and GRP78 in drug-resistant melanomas
}

\author{
Shirish Shenolikar
}

Program in Cardiovascular and Metabolic Disorders, Duke-NUS Graduate Medical School Singapore, Singapore.

\begin{abstract}
$B R A F$ mutations in aggressive melanomas result in kinase activation. BRAF inhibitors reduce $\mathrm{BRAF}^{\mathrm{V} 600 \mathrm{E}}$ tumors, but rapid resistance follows. In this issue of the JCI, Ma and colleagues report that vemurafenib activates ER stress and autophagy in $\mathrm{BRAF}^{\mathrm{V} 600 \mathrm{E}}$ melanoma cells, through sequestration of the ER chaperone GRP78 by the mutant BRAF and subsequent PERK activation. In preclinical studies, treating vemurafenib-resistant melanoma with a combination of vemurafenib and an autophagy inhibitor reduced tumor load. Further work is needed to establish clinical relevance of this resistance mechanism and demonstrate efficacy of autophagy and kinase inhibitor combinations in melanoma treatment.
\end{abstract}

\section{Protein kinases and cancer}

Molecular analyses of human tumors have highlighted the numerous mutations in protein kinase genes that contribute to the development of cancers. For example, the gene encoding BRAF kinase is mutated in more than $60 \%$ of

Conflict of interest: The author's research is supported in part by an Academic Center of Excellence (ACE) Award from GlaxoSmithKline Inc

Citation for this article: J Clin Invest. 2014; 124(3):973-976. doi:10.1172/JCI74609. melanomas, the most aggressive human skin cancer. Indeed, a mutation that results in a single amino acid substitution, V600E, accounts for $90 \%$ of identified $B R A F$ mutations. It is worth noting that this "oncogenic" BRAF (BRAFV600E) is present in many melanocytic nevi, which are benign neoplasias that can persist for decades without transitioning to malignancy (1). The fact that

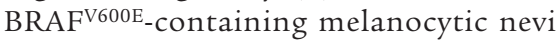
are not intrinsically aggressive hints that additional steps - such as acquisition of further mutations, metabolic reprogramming (2), or alterations in other cellular processes - are required for conversion of the proliferative melanocytes into full-fledged malignant melanomas. This raises the exciting possibility that understanding some of the internal brakes on malignancy will yield novel treatments for melanoma that fails to respond to most current anticancer therapies.

Cancer-promoting mutations frequently result in constitutive activation of the mutant kinase, which has prompted pharmaceutical companies to develop kinase inhibitors to slow or reverse the oncogenic process (3). However, the networks of signaling pathways that control cell growth in normal and cancer cells means that most kinase inhibitors are cytostatic, causing cell cycle arrest rather than cell death. Some kinase inhibitors, however, show spectacular results in eradicating tumor cells. For example, the BCR-ABL tyrosine kinase inhibitor ima- 
A

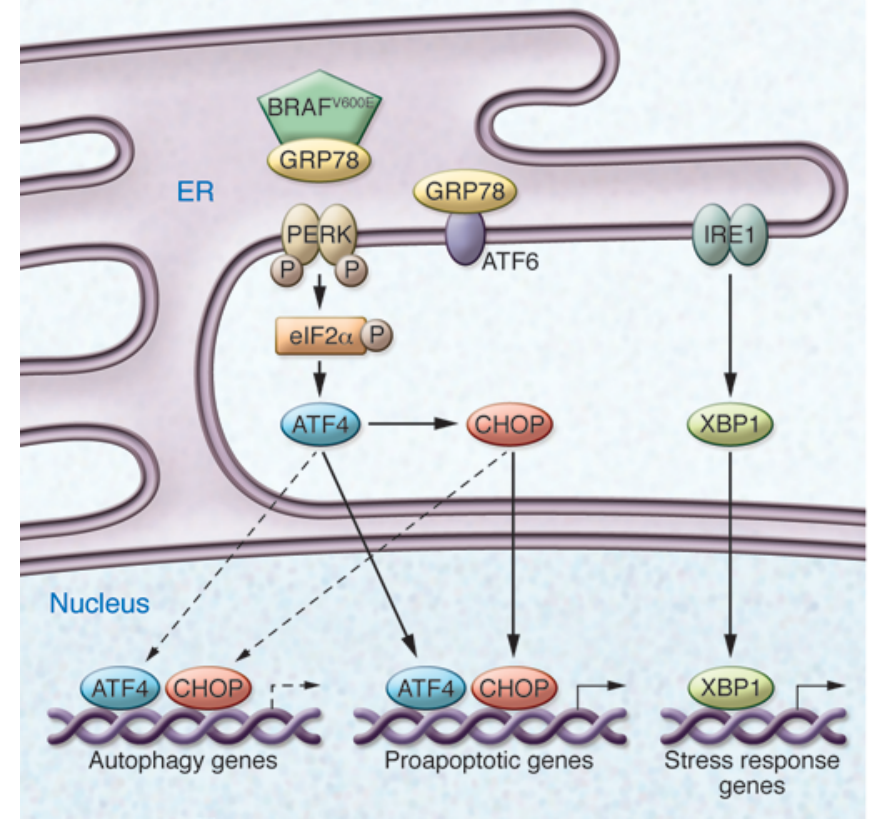

B

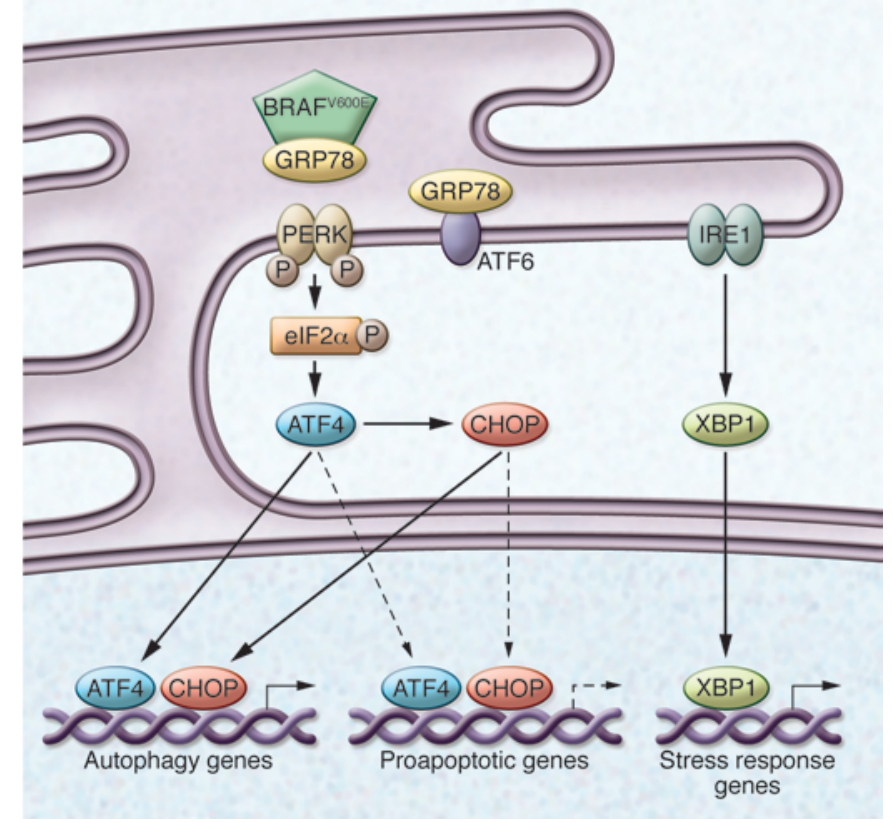

Figure 1

The BRAF inhibitor vemurafenib activates ER stress. Vemurafenib promotes the association of BRAFV600E with GRP78 in the ER in drug-sensitive (A) and drug-resistant (B) melanoma cells. This association displaces GRP78 from PERK, resulting in robust autophosphorylation and kinase activation. The ensuing PERK-mediated phosphorylation of elF2 $\alpha$ initiates a transcriptional and translational cascade that is mediated by expression of the transcription factors ATF4 and CHOP. These nuclear factors either act alone or together to promote the expression of numerous proapoptotic - and, paradoxically, also autophagic (11) - genes. Vemurafenib treatment also results in IRE1 activation and subsequent splicing of the mRNA encoding X-box binding protein 1 (XBP1), a transcription factor that regulates ER-associated protein degradation in response to ER stress. In drug-sensitive melanomas (A), apoptosis prevails, while in drug-resistant tumor cells (B), autophagy may override apoptosis. The factors that shift the balance from apoptosis to autophagy in response to vemurafenib treatment remain unknown, but could include the expression of cellular IAPs or expression of other antiapoptotic genes that contribute to tumor survival in the presence of the BRAF inhibitor. The present findings of Ma et al. (8) suggest that autophagy inhibitors combined with vemurafenib may be an effective therapy for melanoma that would eliminate both drug-sensitive and drug-resistant tumor cells.

tinib elicits greater than $80 \%$ response in the chronic phase of chronic myeloid leukemia (CML). Similarly, the BRAF inhibitor vemurafenib shows dramatic responses in melanoma patients harboring the BRAF ${ }^{V 600 E}$ mutation (4). For both imatinib and vemurafenib, drug resistance emerges rapidly in nearly all patients. Understanding how these drugs instigate cell death and the molecular basis underlying resistance should facilitate future development of improved anticancer therapies.

\section{Kinase inhibitors activate ER stress and induce autophagy}

The combination of an unfavorable microenvironment, a poor nutrient supply, and the energy demands associated with increased protein synthesis in rapidly proliferating cancer cells create ER stress. In melanoma cells, ER stress is reflected by elevated expression of the ER luminal marker glucose-regulated protein 78 (GRP78; also known as BIP) (Figure 1 and ref. 5). Additionally, there is growing awareness that many anticancer therapies induce ER stress, which plays a key role in triggering apoptosis, or programmed cell death, in tumor cells. For instance, vemurafenib has been shown to activate ER stress signaling pathways in melanoma cells, specifically those triggered by the ER membrane-localized sensors PERK, ATF6, and IRE1. In BRAFV600E melanoma cells, knockdown of ATF4, an ER stressinduced transcription factor acting in the PERK pathway, markedly reduced vemurafenib-associated cell death. Conversely, vemurafenib combined with either thapsigargin or tunicamycin, both of which are known inducers of ER stress, enhanced apoptosis in vemurafenibresistant or -insensitive melanoma cells (6). These results suggest that pharmacological enhancers of ER stress, particularly those that stimulate the PERK pathway, may be useful adjuncts to vemurafenib treatment and may overcome the rapidly developing drug resistance seen in melanoma patients.

The transient activation of the PERK, ATF6, and IRE1 ER stress-activated pathways constitutes a normal homeostatic mechanism present in all mammalian cells to cope with unfavorable environmental conditions. In contrast, persistent signaling via the PERK (and IRE1) pathway promotes apoptosis, eliminating damaged or dysfunctional cells and preserving overall tissue function. The PERK pathway also stimulates autophagy to salvage components of the damaged cells that can be used to sustain the remaining cells (Figure 1 and ref. 5). In a cancer setting, autophagy frequently overrides or overcomes ER stress-induced cell death; therefore, therapy-induced ER stress, which is frequently accompanied 
by enhanced autophagy, may contribute to the development of drug resistance. In CML cells, attenuating autophagy in combination with imatinib therapy enhances cell death and even eliminates CML stem cells, which are often difficult to eradicate with imatinib alone (7). In this issue of the JCI, Ma et al. reveal that autophagy is also elevated in melanoma tumor samples from patients treated with the BRAF inhibitors vemurafenib and dabrafenib (8). Autophagy biomarkers were also increased in samples taken after the emergence of drug resistance compared with samples taken prior to treatment. Furthermore, BRAF inhibitor-induced autophagy was preceded by ER stress in both drug-sensitive and -resistant BRAFV600E melanoma cell lines. Importantly, autophagy inhibition enhanced kinase inhibitor-induced cell death both in culture and in a mouse xenograft melanoma model. Together, these findings raise new hopes that a similar drug combination strategy may limit drug resistance and improve the efficacy of current treatment regimens for malignant melanoma.

The work by Ma and colleagues (8) also provides mechanistic insight into the actions of BRAF inhibitors, suggesting that they act upstream of PERK and promote PERK autophosphorylation, which is canonically activated in response to misfolded proteins in the ER. Misfolded proteins displace GRP78, which suppresses PERK kinase activity by binding its ER luminal domain. GRP78 displacement promotes PERK dimerization and autophosphorylation, increasing PERK activity, as evidenced by the enhanced phosphorylation of the eukaryotic initiation factor eIF $2 \alpha$ and the subsequent attenuation of mRNA translation. Remarkably, $\mathrm{Ma}$ and colleagues found that BRAF inhibitors enhanced GRP78 binding to BRAF $^{\mathrm{V} 600 \mathrm{E}}$ within the ER lumen, and this interaction correlated with a reduction in GRP78 binding to PERK (8). These data suggest that in the presence of BRAF inhibitors, BRAFV600E outcompetes PERK for GRP78 binding, thereby explaining the activation of PERK by these drugs. The interaction between GRP78 and BRAFV600E is somewhat surprising, because GRP78 is a well-recognized ER luminal chaperone and possesses a C-terminal KDEL ER retention sequence. However, some studies have suggested that GRP78 can be translocated to the cytosol and plasma membrane in response to ER stress (9). In contrast, BRAF is primarily cytoplasmic, although one report suggests that a small fraction of BRAF in thyroid cancer cells may be associated with mitochondria. To date, the ER localization of BRAF - either WT or mutant forms of the protein - has not been reported. Substantially less BRAFV600E bound to GRP78 in the absence of BRAF inhibitors in the cytoplasm.

\section{Conclusions and future directions}

An interpretation of the work of Ma et al. (8) might be that BRAF inhibitors promote the translocation of BRAFV600E to ER, a major site of GRP78 in cells, thereby enhancing the BRAF/GRP78 interaction. A puzzling aspect is the apparent finding that PERK was preferentially or selectively activated after GRP78 binding to BRAF. The ER luminal domains of the three ER stress sensors, PERK, ATF6, and IRE1, are highly homologous, and all three proteins bind GRP78; therefore, it would be expected that the sequestration of GRP78 by BRAFV600E might activate all three ER stress-signaling pathways. On the other hand, earlier studies have shown that GRP78, which is induced by ATF6, is decreased in melanoma cells after vemurafenib exposure (6). At this stage, not only is it unclear how BRAF inhibitors promote BRAF entry into the $\mathrm{ER}$, but it also remains to be determined how and why the sequestration of GRP78 by BRAF preferentially activates PERK. Another complicating factor is the recent finding that vemurafenib displaces BRAFV600E from the HSP90/CDC37 chaperone/ cochaperone complex (10). The displaced $\mathrm{BRAF}^{\mathrm{V} 600 \mathrm{E}}$ is unstable and is degraded by the proteasome, which suggests that this kinase inhibitor achieves some of its therapeutic effect by reducing cellular BRAF ${ }^{\mathrm{V} 600 \mathrm{E}}$ levels. Whereas these data indicate that vemurafenib decreases both GRP78 and BRAF ${ }^{\mathrm{V} 600 \mathrm{E}}$ levels in melanoma cells, Ma and colleagues demonstrated an enhanced association of GRP78 and $\mathrm{BRAF}^{\mathrm{V} 600 \mathrm{E}}$ in the presence of this BRAF inhibitor (8). Thus, more work is clearly needed to fully understand these differing effects of the BRAF inhibitor and their impact on ER stress in vemurafenibtreated cells.

In summary, recent studies highlight the ability of anticancer therapies to activate ER stress, which may be critical for the induction of apoptosis in tumor cells; however, therapy-induced ER stress, specifically that associated with PERK activation, also promotes cancer cell survival via activation of autophagy (11). Other studies suggest that the PERK pathway also enhances the expression of inhibitors of apoptosis (IAPs), which are frequently elevated in melanoma cells and may also contribute to cancer cell survival or drug resistance. An emerging strategy for a more effective anticancer therapy is to combine kinase inhibitors with autophagy inhibitors to achieve the best clinical outcomes for CML and melanoma. The inclusion of IAP-inhibiting compounds to enhance apoptosis may also help reduce the rapid resistance to the frontline drugs. It will be exciting to see just how this new strategy plays out in the coming years and whether it offers new hope for the eradication of the aforementioned cancers.

\section{Acknowledgments}

The author's research is supported by grants from National Medical Research Council and Biomedical Research Council of Singapore as well as by a Duke/ Duke-NUS Collaborative Research Grant. Additional funding was provided through a GlaxoSmithKline Academic Center of Excellence (ACE) Award.

Address correspondence to: Shirish Shenolikar, Program in Cardiovascular and Metabolic Disorders, Duke-NUS Graduate Medical School Singapore, 8 College Road, Singapore 169857, Singapore. Phone: 65.6516.2588; Fax: 65.6220.8161; E-mail: shirish.shenolikar@duke-NUS.edu.sg.

1. Takata M, Saida T. Genetic alterations in melanocytic tumors. J Dermatol Sci. 2006;43(1):1-10.

2. Pollack M. Targeting oxidative phosphorylation: why, when, and how. Cancer Cell. 2013;23(3):263-264.

3. Zhang J, Yang PL, Gray NS. Targeting cancer with small molecule kinase inhibitors. Nat Rev Cancer. 2009;9(1):28-39.

4. Chapman $P B$, et al. Improved survival with vemurafenib in melanoma with BRAF V600E mutation. N Engl J Med. 2011;364(26):2507-2516.

5. Luo B, Lee AS. The critical roles of endoplasmic reticulum chaperones and unfolded protein response in tumorigenesis and anti-cancer therapies. Oncogene. 2013;32(7):805-818.

6 . Beck D, et al. Vemurafenib potently induces endoplasmic reticulum stress-mediated apoptosis in BRAFV600E melanoma cells. Sci Signal. 2013; 6(260):ra7.

7. Galavotti S, et al. Targeting autophagy potentiates tyrosine kinase inhibitor-induced cell death in Philadelphia chromosome-positive cells, including primary CML stem cells. J Clin Invest. 2009; 119(5):1109-1123.

8. Ma X-H, et al. Targeting ER stress-induced autophagy overcomes BRAF inhibitor resistance in mela- 
noma. J Clin Invest. 2014;124(3):1406-1417.

9. Ni M, Zhang Y, Lee AS. Beyond the endoplasmic reticulum: atypical GRP78 in cell viability, signalling and therapeutic targeting. Biochem J.
2011;434(2):181-188

10. Polier S, Samant RS, Clarke PA, Workman P, Prodromou C, Pearl LH. ATP -competitive inhibitors block protein kinase recruitment to the Hsp90-
Cdc37 system. Nat Chem Biol. 2013;9(5):307-312.

11. B'chir W, et al. The eIF $2 \alpha /$ ATF4 pathway is essential for stress-induced autophagy gene expression. Nucleic Acids Res. 2013;41(16):7683-7699.

\title{
Dynamin 2 the rescue for centronuclear myopathy
}

\author{
Alexis R. Demonbreun ${ }^{1}$ and Elizabeth M. McNally, ${ }^{1,2}$
}

${ }^{1}$ Department of Medicine and 2Department of Human Genetics, University of Chicago, Chicago, Illinois, USA.

\begin{abstract}
Centronuclear myopathy is a lethal muscle disease. The most severe form of the disease, $X$-linked centronuclear myopathy, is due to mutations in the gene encoding myotubularin (MTM1), while mutations in dynamin 2 (DNM2) and amphiphysin 2/BIN1 (AMPH2) cause milder forms of myopathy. MTM1 is a lipid phosphatase, and mutations that disrupt this activity cause severe muscle wasting. In this issue of the JCI, Cowling and colleagues report on their finding of increased DNM2 levels in human and mouse muscle with MTM1 mutations. Partial reduction of Dnm2 in mice harboring Mtm1 mutations remarkably rescued muscle wasting and lethality, and this effect was muscle specific. DNM2 regulates membrane trafficking through vesicular scission, and it is presumed that reducing this activity accounts for improved outcome in X-linked centronuclear myopathy.
\end{abstract}

\section{Defective membranes in centronuclear myopathies}

Centronuclear myopathy (CNM) is an inherited muscle-wasting disease with onset in infancy or childhood. Muscle biopsies from CNM patients characteristically show enlarged and centrally placed nuclei throughout the muscle, and clinically, CNM-associated mutations may result in weakened respiratory musculature, leading to early death without supportive care. Three main forms of CNM are attributable to mutations in myotubularin (MTM1), amphiphysin 2 (AMPH2), and dynamin 2 (DNM2), which all encode membraneassociated proteins that potentially act in muscle at the transverse tubules (T-tubules), membrane invaginations specialized for calcium handling (Figure 1 and ref. 1). The most common and severe form of CNM is X-linked CNM (XLCNM), referred to as myotubular myopathy. XLCNM is clinically evident at birth, with patients presenting with hypotonia and respiratory compromise. Skeletal muscles biopsies from XLCNM patients show abnormal T-tubules, including defective triads, struc-

Conflict of interest: The authors have declared that no conflict of interest exists.

Citation for this article: J Clin Invest. 2014; 124(3):976-978. doi:10.1172/JCI74434. tures critical for excitation-contraction coupling. XLCNM is the result of mutations in MTM1, which encodes a ubiquitously expressed phosphoinositide 3-phosphatase that functions to dephosphorylate PI3P and phosphatidylinositol-3,4-bisphosphate, two phospholipids essential for membrane sorting and signaling (2). MTM1 is required for normal endocytosis, receptor degradation, and early endosome maturation (1).

Mice harboring a deletion of Mtm1 (Mtm1-/y mice) recapitulate human disease and display many characteristic XLCNMassociated features, including muscle weakness, centralized nuclei, and muscle atrophy (3). Mtm $1^{-/ y}$ mice exhibit muscle atrophy, become weak at 3 to 4 weeks of age, and have a greatly reduced life expectancy of only 6 to 12 weeks (3). The role of MTM1 in human muscle has also been effectively modeled in zebrafish using morpholinos to reduce $m t m 1$ expression (MTM MO) (4). MTM $\mathrm{MO}$ fish, like XLCNM patients and $\mathrm{Mtm}^{-/} \mathrm{H}^{-1}$ mice, develop weakness and muscle atrophy. MTM MO fish display accumulation of PI3P, especially surrounding muscle nuclei, and T-tubule defects. The phenotypes observed in MTM MO fish provide further support that MTM1 is critical for the normal biogenesis and maintenance of membrane structures within muscle (4). Because of its broad role as a lipid phosphatase, the indispensable targets of MTM1's enzymatic action are not fully known.

DNM2 is a ubiquitously expressed GTPase that has been implicated in multiple cellular functions, including endocytosis, membrane scission, and cytoskeletal remodeling (5). Dynamins assemble as rings around membrane tubules where they are thought to actively "pinch off" membranes (5). Complete loss of Dnm2 results in embryonic lethality in mice (6). In humans, CNM is caused by dominant DNM2 mutations (7), and overexpression of a CNM-linked DNM2 mutation (R465W) in mouse muscle results in myopathic features, including centralized nuclei, muscle atrophy, and deformed T-tubules (8). Interestingly, overexpression of normal DNM2 in mouse models also produces some of these same features, consistent with the model that increased DNM2 activity contributes to CNM (8). Increased DNM2 activity may lead to excessive membrane scission and pruning, giving the appearance of excessive membrane accumulation around nuclei or at T-tubules.

\section{Genetic reduction of DNM2 in a CNM model}

In this issue of the JCI, Cowling and colleagues described elevated protein levels of DNM2 in $M t m 1^{-/ y}$ animals and in muscle biopsies from human XLCNM patients (9). To target the increased levels of DNM2 found in $M t m 1^{-/ y}$ animals, mice heterozygous for Dnm2 were crossed with $M t m 1^{-1 y}$ mice. The in vivo reduction of Dnm2 corrected some histological abnormalities in muscle, but dramatically extended life expectancy from 6 to 12 weeks to beyond one year. To demonstrate that this effect was muscle intrinsic, Cowling and colleagues generated $M t m 1^{-/ y}$ mice in which $D n m 2$ was specifically reduced in skeletal muscle. Reduction of Dnm2 in skeletal muscle alone after disease onset was sufficient to reduce pathology and 